\author{
UNIVERSIDADE DE SÃO PAULO \\ ESCOLA DE ENFERMAGEM DE RIBEIRÃO PRETO
}

\title{
FATORES DE RISCO PARA AS DOENÇAS \\ CARDIOVASCULARES EM TRABALHADORES DE UMA \\ DESTILARIA DO INTERIOR PAULISTA
}

\author{
Manuel Simão
}

Ribeirão Preto

2001 


\author{
UNIVERSIDADE DE SÃO PAULO \\ ESCOLA DE ENFERMAGEM DE RIBEIRÃO PRETO
}

\title{
FATORES DE RISCO PARA AS DOENÇAS CARDIOVASCULARES EM TRABALHADORES DE UMA DESTILARIA DO INTERIOR PAULISTA
}

\author{
Manuel Simão
}

\begin{abstract}
Dissertação apresentada ao Programa de Pósgraduação - Área Enfermagem Fundamental - nível Mestrado - do departamento de Enfermagem Geral e Especializada da Escola de Enfermagem de Ribeirão Preto da Universidade de São Paulo, como requisito à obtenção do grau de Mestre em Enfermagem, vinculada à linha de investigação Processo de Cuidar do Adulto com Doenças Agudas e Crônicas Degenerativas
\end{abstract}

Orientadora: $\operatorname{Prof}^{\mathrm{a}} \mathrm{Dr}^{\mathrm{a}}$ Maria Suely Nogueira

\author{
RIBEIRÃO PRETO
}

2001 
Ficha Catalográfica elaborada pela Biblioteca Central Campus USP - Ribeirão Preto

Simão, Manuel
Fatores de risco para as doenças cardiovasculares em
trabalhadores de uma destilaria do interior paulista. Ribeirão
Preto, 2001.
86p.: il.; 30cm.
Dissertação de Mestrado, apresentada à Escola de Enfermagem de
Ribeirão Preto/USP. Departamento de Enfermagem Geral e Especializada.
Área: Enfermagem Fundamental.
Orientador: Nogueira, Maria Suely
1. Doenças cardiovasculares. 2. Fatores de risco. 3.
hipertensão arterial


Aos meus pais, Simão e Isabel, pelo início de tudo. Com seu exemplo ensinaram-me a ser persistente e confiante.

Ao meu filho Gildo, à sua mãe Maria de Fátima e a todos meus familiares que com paciência, sempre compreenderam a vivência à longa distância, por vários anos.

$A$ vocês ofereço o meu afeto e esta conquista 
À REPÚBLICA DE ANGOLA, NAÇÃO A QUAL PERTENÇO,

MEU MUITO OBRIGADO 


\section{AGRADECIMENTOS}

À República Federativa do Brasil pela hospitalidade aprazível, desde o período da graduação.

À Prof $^{a} \operatorname{Dr}^{\mathrm{a}}$ Maria Suely Nogueira, minha orientadora, pela generosidade, incentivo, confiança, apoio e tranqüilidade em todos os momentos desta trajetória.

À $\operatorname{Dr}^{\mathrm{a}}$ Miyeko Hayashida, pela disponibilidade em ajudar desde o início do trabalho e pelas valiosas contribuições para o êxito deste trabalho.

Ao Prof Dr Evandro José Cesarino, pelas suas valiosas sugestões visando o aprimoramento deste trabalho.

À Escola de Enfermagem de Ribeirão Preto da Universidade de São Paulo e aos docentes desta instituição, pela inestimável contribuição para a minha formação, desde a graduação.

A todos os funcionários da Escola de Enfermagem de Ribeirão Preto da Universidade de São Paulo, pelo carinho, cordialidade e apoio.

Ao Ministério da Saúde de Angola, pelo auxílio financeiro concedido para compra de minha passagem aérea para o Brasil.

A todos os enfermeiros licenciados em enfermagem de Angola, pelo incentivo, força e coragem para que eu realizasse esta etapa.

Sou grato também:

Aos colegas da Escola Técnica Profissional de Saúde do Lubango, por terem entendido a necessidade da minha ausência naquela instituição. 
Aos colegas Pós-Graduandos da EERP-USP e amigos da casa 13, pelos momentos agradáveis de convivência.

À Carla e Giovanna, pelo apoio na tradução e redação dos textos em inglês e espanhol.

As bibliotecárias Cristina e Bernadete, pelo apoio na revisão bibliográfica.

Ao Ademir Dorascenzi, pelo valioso apoio que tornou possível a realização deste estudo.

À Elizangela Sordi e Raquel Palhares, pela contribuição na realização deste estudo.

À Direção da destilaria Santa Inês, por acreditar na seriedade deste trabalho e por permitir a realização da coleta de dados e pelo carinho dispensado à minha pessoa.

Ao Dr José Guilherme Barbosa, Sra Neli Aparecida Tonielo Barbosa, Sra Marta Aparecida de Sousa, Sra Ivone Aparecida Silva, $\mathrm{Sr}^{\mathrm{a}}$ Olinda Tereza Guidugli e Sr Angenor Luiz, pelo carinho com que me acolheram na destilaria Santa Inês, por ocasião da coleta de dados.

Às Senhoras Ana Maria, Célia Regina, Conceição e Lorelay, pelo carinho dispensado.

Aos trabalhadores que, de maneira simples e acolhedora, tornaram possível a coleta dos dados deste trabalho.

Aos amigos Sebastião, Duarte, Arlindo, Roselyne, Regina, Amasília, pelo estímulo de força para a realização deste trabalho.

A todos que mesmo não tenho seus nomes citados, direta ou indiretamente participaram e contribuíram nas etapas vivenciadas para a realização desta dissertação. 
A Agência Sueca para o Desenvolvimento Internacional (ASDI), pelo financiamento do curso de graduação em enfermagem o que me possibilitou chegar até aqui.

À Coordenação de Aperfeiçoamento de Pessoal de Nível Superior (CAPES), pelo apoio financeiro concedido. 


\section{S U M Á R I O}

ABREVIATURAS UTILIZADAS

LISTA DE TABELAS

RESUMO

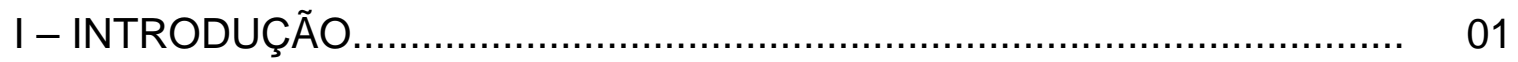

1.1. Referencial teórico.................................................. 13

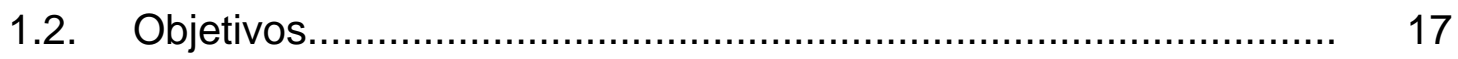

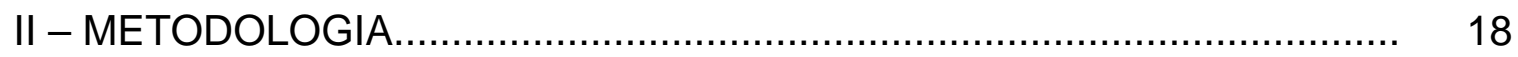

2.1. Delineamento do estudo.................................................. 18

2.2. Local de estudo....................................................... 18

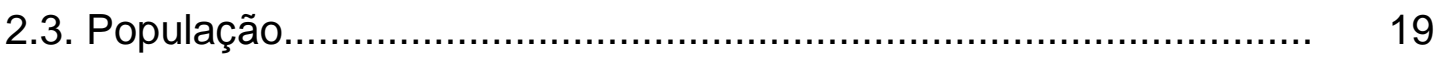

2.4. Procedimento para coleta de dados......................................... 20

2.4.1. Instrumento de coleta de dados................................... 20

2.4.2. Pré-teste........................................................ 21

2.4.3. Operacionalização da coleta de dados.............................. 22

2.5. Procedimento de análise dos dados...................................... 25

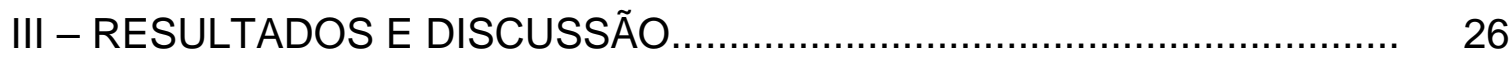

3.1. Caracterização da população e dados sobre a biologia humana...... 26

3.2. Dados relacionados ao meio ambiente................................. 37

3.3. Dados relacionados ao estilo de vida.................................. 47

3.4. Dados relacionados ao atendimento de saúde........................... 71

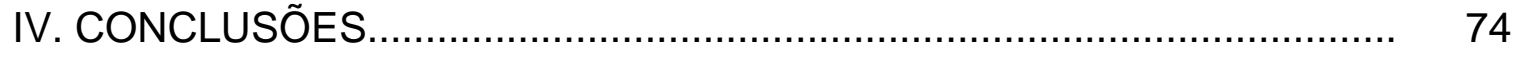

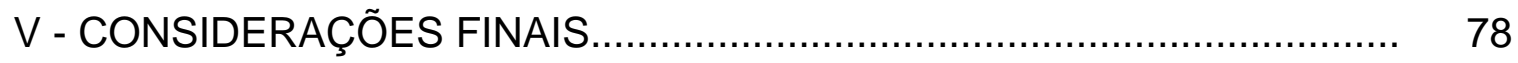

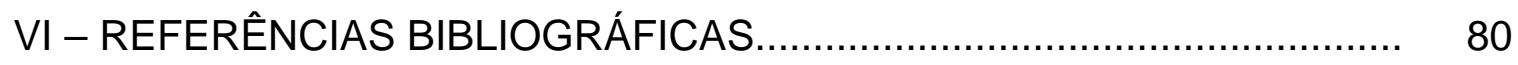

SUMMARY

RESUMEN

ANEXOS 
AVC: Acidente vascular cerebral

CNS: Conselho Nacional de Saúde

CO: Monóxido de carbono

DAC: Doença arterial coronária

DCV: Doença cardiovascular

DCVs: Doenças cardiovasculares

DIC: Doença isquêmica do coração

DICs: Doenças isquêmicas do coração

DM: Diabetes mellitus

HA: Hipertensão arterial

HAS: Hipertensão arterial sistêmica

HDL: Lipoproteínas de alta densidade

IMC: Î́ndice de massa corporal

LDL: Lipoproteínas de baixa densidade

mmHg: Milímetros de mercúrio

OMS: Organização Mundial da Saúde

OPS: Organização Pan-Americana de Saúde

PA: Pressão arterial

PAD: Pressão arterial diastólica

PAS: Pressão arterial sistólica

VLDL: Lipoproteínas de muito baixa densidade 


\section{LISTA DE TABELAS}

Tabela 1: Distribuição dos trabalhadores de uma destilaria segundo a variável idade em anos. Ribeirão Preto, 2001...................................................26

Tabela 2: Distribuição dos trabalhadores de uma destilaria, segundo o Índice de Massa Corporal (IMC). Ribeirão Preto, 2001.......................................27

Tabela 3: Distribuição dos trabalhadores de uma destilaria segundo a idade em anos, por faixa etária e Índice de Massa Corporal. Ribeirão Preto, 2001....28

Tabela 4: Distribuição dos trabalhadores de uma destilaria segundo a idade em anos, por faixa etária e os valores da pressão arterial sistólica (PAS). Ribeirão Preto, 2001. 29

Tabela 5: Distribuição dos trabalhadores de uma destilaria segundo a idade por faixa etária e os valores de pressão arterial diastólica (PAD). Ribeirão Preto, 2001 29

Tabela 6: Distribuição dos trabalhadores de uma destilaria segundo o Índice de Massa Corporal (IMC) e os valores de pressão arterial sistólica (PAS). Ribeirão Preto, 2001 .30

Tabela 7: Distribuição dos trabalhadores de uma destilaria segundo o Índice de Massa Corporal (IMC) e os valores da pressão arterial diastólica (PAD). Ribeirão Preto, 2001 30

Tabela 8: Distribuição dos trabalhadores de uma destilaria segundo a etnia e os valores de pressão arterial sistólica (PAS). Ribeirão Preto, 2001

Tabela 9: Distribuição dos trabalhadores de uma destilaria segundo a etnia e os valores da pressão arterial diastólica (PAD). Ribeirão Preto, 2001..............31

Tabela 10: Distribuição dos trabalhadores de uma destilaria segundo o grau de parentesco indicado para antecedente familiar com hipertensão arterial sistêmica (HAS). Ribeirão Preto, 2001 .35

Tabela 11: Distribuição dos trabalhadores de uma destilaria segundo a categoria de ocupações desenvolvidas na empresa. Ribeirão Preto, 2001 38

Tabela 12: Distribuição dos trabalhadores de uma destilaria segundo a categoria de ocupações desenvolvidas na empresa e os valores de pressão arterial sistólica (PAS). Ribeirão Preto, 2001. .38

Tabela 13: Distribuição dos trabalhadores de uma destilaria segundo a categoria de ocupações desenvolvidas na empresa e os valores de pressão arterial diastólica (PAD). Ribeirão Preto, 2001 
Tabela 14: Distribuição dos trabalhadores de uma destilaria segundo o tempo em anos de atividade na empresa. Ribeirão Preto, 2001

Tabela 15: Distribuição dos trabalhadores de uma destilaria segundo a jornada de trabalho. Ribeirão Preto, 2001

Tabela 16: Distribuição dos trabalhadores de uma destilaria segundo a renda familiar em salários-mínimos. Ribeirão Preto, 2001.

Tabela 17: Distribuição dos trabalhadores de uma destilaria segundo a renda familiar em salários-mínimos e os valores da pressão arterial sistólica (PAS). Ribeirão Preto, 2001 .43

Tabela 18: Distribuição dos trabalhadores de uma destilaria segundo a renda familiar em salários-mínimos e a pressão arterial diastólica (PAD). Ribeirão Preto, 2001 .43

Tabela 19: Distribuição dos trabalhadores de uma destilaria segundo o número de pessoas que compõem a família. Ribeirão Preto, 2001 44

Tabela 20: Distribuição dos trabalhadores de uma destilaria segundo o número de pessoas da família que exercem atividade remunerada. Ribeirão Preto 2001. .44

Tabela 21: Distribuição dos trabalhadores de uma destilaria segundo a contribuição dos membros da família para a renda familiar em saláriosmínimos. Ribeirão Preto, 2001 45

Tabela 22: Distribuição dos trabalhadores de uma destilaria segundo o estado civil. Ribeirão Preto, 2001 46

Tabela 23: Distribuição dos trabalhadores de uma destilaria segundo o Estado de procedência. Ribeirão Preto, 2001.

Tabela 24: Distribuição dos trabalhadores de uma destilaria segundo os tipos de atividades físicas praticados. Ribeirão Preto, 2001

Tabela 25: Distribuição dos trabalhadores de uma destilaria segundo a freqüência e duração de atividades físicas praticadas. Ribeirão Preto, 2001 49

Tabela 26: Distribuição dos trabalhadores de uma destilaria segundo a prática de atividade física e o Índice de Massa Corporal. Ribeirão Preto, 2001. .50

Tabela 27: Distribuição dos trabalhadores de uma destilaria segundo a realização de atividade física e valores de pressão sistólica (PAS). Ribeirão Preto, 2001. 
Tabela 28: Distribuição dos trabalhadores de uma destilaria segundo a realização de atividade física e os valores de pressão arterial diastólica (PAD). Ribeirão Preto, 2001

Tabela 29 - Distribuição dos trabalhadores de uma destilaria segundo o tipo e a quantidade de cigarro e os valores de pressão arterial sistólica (PAS). Ribeirão Preto, 2001. .52

Tabela 30: Distribuição dos trabalhadores de uma destilaria segundo o tipo e a quantidade de cigarro e os valores de pressão arterial diastólica (PAD). Ribeirão Preto, 2001.

Tabela 31: Distribuição dos trabalhadores de uma destilaria segundo o tipo de bebida alcoólica consumida. Ribeirão Preto, 2001. 55

Tabela 32: Distribuição dos trabalhadores de uma destilaria segundo o tipo de bebida alcoólica consumida e os valores de pressão arterial sistólica (PAS). Ribeirão Preto, 2001 58

Tabela 33: Distribuição dos trabalhadores de uma destilaria segundo o tipo de bebida alcoólica consumida e os valores de pressão arterial diastólica (PAD). Ribeirão Preto, 2001 .58

Tabela 34: Distribuição dos trabalhadores de uma destilaria segundo a freqüência do consumo de alimentos. Ribeirão Preto, 2001. 65

Tabela 35: Distribuição dos trabalhadores de uma destilaria segundo o conhecimento acerca dos fatores de risco para as doenças cardiovasculares. Ribeirão Preto, 2001................................................70

Tabela 36 - Distribuição dos trabalhadores de uma destilaria segundo o tipo de patologia sob tratamento. Ribeirão Preto, 2001 .73 


\section{RESUMO}

\section{FATORES DE RISCO PARA AS DOENÇAS CARDIOVASCULARES EM TRABALHADORES DE UMA DESTILARIA DO INTERIOR PAULISTA}

Desenvolvemos estudo entre trabalhadores de uma destilaria do interior paulista com o objetivo de identificar o perfil de trabalhadores do sexo masculino quanto aos fatores de risco cardiovasculares. O referencial teórico foi o Modelo de "Campo de Saúde" que compõe os elementos de biologia humana, meio ambiente, estilo de vida e organização dos serviços de saúde. A população estudada constituiu-se de 123 trabalhadores, com idade entre 18 e 71 anos, que desenvolviam atividades no setor da indústria. Os dados foram obtidos através da entrevista individual feita pelo pesquisador na própria destilaria onde os mesmos atuavam. Ao término de cada entrevista procedeu-se a verificação da pressão arterial pelo método indireto, em seguida o exame antropométrico que constituiu-se da medida de peso e altura. Os níveis tensionais foram obtidos através de duas medidas com utilização do aparelho oscilométrico da marca Dixtal (modo automático) e manguitos de bolsa de borracha compatíveis com a circunferência braquial do indivíduo. Com relação a biologia humana, 10,6\% dos trabalhadores apresentou obesidade grau I, 17,0\% obesidade grau II; em 11,4\% dos indivíduos identificamos valores de pressão arterial sistólica $\geq$ $140 \mathrm{mmHg}$ e em 12,2\% com pressão arterial diastólica $\geq 90 \mathrm{mmHg}$; quanto aos antecedentes familiares, $32,5 \%$ referiram história familiar positiva de doença hipertensiva, 26,0\% de acidente vascular cerebral e 27,6 de diabetes melitus. Quanto ao meio ambiente, $60,2 \%$ possuíam o $1^{\circ}$ grau incompleto, 49,6\% eram casados e $97,6 \%$ tinham o único emprego. No tocante ao estilo de vida, $40,7 \%$ referiram realizar algum tipo de atividade física, 16,3\% eram fumantes, 65,9\% indicaram consumo de bebida alcoólica e 49,6\% referiram ingerir alimentos preparados com muito sal. Em relação ao atendimento de saúde todos trabalhadores participantes no estudo possuíam convênio médico, além daquele oferecido pela própria empresa. Os dados revelam existência de hábitos autocriados que podem ser modificados e que se constituem em fatores de risco cardiovascular; realçamos a necessidade de promoção de ações educativas visando à prevenção dessas doenças.

Palavras chave: doenças cardiovasculares, fatores de risco, hipertensão arterial 


\section{SUMMARY}

\section{RISK FACTORS OF CARDIOVASCULAR DISEASES IN WORKERS OF A DISTILLERY IN THE INTERIOR OF SÃO PAULO STATE}

This study was developed among workers of a distillery in the interior of São Paulo State aiming at identifying the profile of male workers with regard to risk factors of cardiovascular diseases. The theoretical framework was based on the "Health Field" Model, which composes the elements of human biology, environment, lifestyle and organization of health services. The studied population consisted of 123 workers aged 18 to 71 years who performed their activities in the factory. Data were obtained by means of individual interviews conducted by the researcher in the facilities of the distillery where the subjects worked. At the end of each interview, each individual's arterial pressure was measured by means of the indirect method, which was followed by the assessment of their anthropometric measures, namely height and weight. Tension levels were obtained through two measurements with the use of an oscillometric device (automatic mode) manufactured by Dixtal and rubber-bag cuffs which were compatible with the individual's brachial circumference. With regard to human biology, $10.6 \%$ of the workers presented level-1 obesity and $17 \%$ had level-2 obesity, $11.4 \%$ of the individuals, systolic arterial pressure values were found to be $\geq 140 \mathrm{mmHG}$ and in $12.2 \%$ diastolic arterial pressure values were found to be $\geq$ $90 \mathrm{mmHG}$. Concerning their family history, 32,5\% reported a family history with the presence of hypertensive disease consisting of cerebral vascular accidents (26.0\%) and diabetes mellitus (27.6\%). As to the environment, $60.2 \%$ had incomplete elementary school education, $49.6 \%$ were married and $97.6 \%$ had only one job. Concerning lifestyle, $40.7 \%$ reported the habit to perform some type of physical activity, $16.3 \%$ were smokers, $65.9 \%$ indicated the consumption of alcoholic beverages and $49.6 \%$ reported to ingest food prepared with a high amount of salt. As to healthcare, all the workers participating in the study had a medical insurance in addition to that provided by the company. Data showed the existence of self-created habits which consisted in risk factors of cardiovascular diseases. Since such habits can be modified, the need to promote educational action aiming at the prevention of such diseases is emphasized.

Key Words: Cardiovascular disease, factors risk, hypertension arterial 


\section{RESUMEN}

FACTORES DE RIESGO EN LAS ENFERMEDADES CARDIOVASCULARES DE TRABAJADORES EN UNA DESTILARIA DEL INTERIOR PAULISTA

Desenvolvemos estudio entre trabajadores de una destilaría del interior paulista con el objetivo identificar el perfil de trabajadores del sexo masculino cuanto a los factores de riesgos cardiovasculares. El referencial teórico fue el Modelo de "Campo de Salud" que compone los elementos biología humana, medio ambiente, estilo de vida y organización de los servicios de salud. La populación estudiada se constituyo de 123 trabajadores, con edad entre 18 y 71 años, que desenvolvían actividades en el sector de industria. Los datos fueron obtenidos a través de entrevista individual hecha por el investigador en la propia destilaría donde los mismos actuaban. Al final de cada entrevista se procedió a la verificación de la presión arterial por el método indirecto, enseguida el examen antropométrico que se constituye por la medida de peso y altura. Los niveles de tensión fueron obtenidos a través de dos medidas con utilización del aparato oscilo métrico da marca Dixtal (modo automático) y manguitos de bolsa de goma compatibles con la circunferencia braquial del individuo. Con relación a biología humana, $10,6 \%$ de los trabajadores presentó obesidad grado I, 17,0\% obesidad grado II; en 11,4\% de los individuos identificamos valores de presión arterial sistólica $\geq 140 \mathrm{mmHg}$ y en $12,2 \%$ con presión arterial diastólica $\geq 90 \mathrm{mmHg}$; cuanto a los antecedentes familiares, $32,5 \%$ refirieron historia familiar de enfermedad hipertensiva, $26,0 \%$ de accidente cerebro vascular y $27,0 \%$ de diabetes mellitus. Cuanto al medio ambiente, $60,2 \%$ poseían el $1^{\circ}$ grado incompleto, 49,6\% eran casados y $97,6 \%$ tenían el único empleo. A lo que se refiere al estilo de vida, 40,7\% refirieron realizar algún tipo de actividad física, 16,3\% eran fumantes, 65,9\% indicaron consumo de bebida alcohólica y 49,6\% refirieron ingerir alimentos preparados con mucha sal. En relación con el atendimiento de salud todos trabajadores participantes en el estudio poseían convenio médico, además de aquel ofrecido por la propia empresa. Los datos revelan existencia de hábitos auto criados que pueden ser modificados y que se constituyen en factores de riesgo cardiovascular; realzamos la necesidad de promoción de acciones educativas visando la prevención de esas enfermedades.

Palabras clave: enfermedad cardiovascular, factores de riesgo, hipertensión arterial 